\title{
Treatment of lupus nephritis - past, present and (near) future
}

David Navarro

Nephrology Department, Centro Hospitalar Universitário de Lisboa Central E.P.E., Hospital Curry Cabral, Lisbon, Portugal

\section{ABSTRACT}

The management of lupus nephritis is challenging for most nephrologists. Many trials have brought only a certain degree of evidence to the diagnosis and treatment of this disease, and many questions remain unanswered. In this review, we will explore the path traveled so far and the steps that are likely to happen in the near future.

Keywords: Lupus nephritis, glomerular disease, systemic erythematosus lupus, immunosuppression

\section{INTRODUCTION}

Systemic lupus erythematosus (SLE) represents an immune deregulation, which emerges from the complex interaction of genetic predisposition with environmental factors, such as ultraviolet light exposure or hormonal factors - which in turn partially justifies the higher incidence in women of childbearing age. This chronic autoimmune disease can affect many organs, including the skin, joints, heart, central nervous system and kidneys, the latter two particularly impactful in terms of morbidity and mortality. ${ }^{1}$

Diagnosis of SLE can be challenging. While improvements have been made in the understanding of SLE mechanisms, their clinical translation is not straightforward. The diagnosis is achieved through a combination of clinical manifestations and immunological laboratory tests, but there are no clear-cut diagnostic criteria for SLE. Classification criteria - such as the one provided by the American College of Rheumatology or by the SLICC (Systemic Lupus International Collaborating Clinics) - were created with the purpose of providing standardized inclusion criteria in SLE-related trials. They have been used as diagnostic criteria, but fail to capture the myriad of possible manifestations, and are generally of more practical value for patients with advanced SLE. In other words, SLE's clinical heterogeneity and subtleties are hard to identify and quantify, resulting in a delay in diagnosis and initiation of treatment.

It is estimated that $60-80 \%$ of SLE patient will have renal involvement. Lupus nephritis ( $L N$ ) is one of the most serious complications of SLE, typically manifested by an increase in serum creatinine or the development of hematuria and/or proteinuria. Left untreated, it may progress towards end-stage renal disease (ESRD) requiring dialysis or renal transplantation, with an incidence of $10 \%$ within 10 years of diagnosis of SLE. Mortality rates are about three times higher in SLE patients and renal involvement is the most important predictor of mortality in SLE activity indexes. ${ }^{2}$ While serum biomarkers are helpful in the diagnosis of SLE, kidney biopsy plays, for the foreseeable future, a central role not only in the diagnosis but also in the management of LN altogether. Kidney biopsy can reliably differentiate patients who benefit from immunosuppression from those who do not - be it because of non-proliferative disease or because of extensive fibrosis, a synonym of poor renal prognosis. It is yet not without its flaws, namely the fact that it is prone to the bias of sampling miss, and that it is merely a snapshot of a dynamic disease characterized by periods of remission and flares. These shortcomings call for repeat protocol kidney biopsies, which are increasingly perceived as a useful tool for nephrologists who take care of LN patients.

In this review, we will discuss the management of the different subtypes of lupus nephritis, focusing on the use of immunomodulatory treatment where appropriate, based on the current consensus on findings and interpretation of kidney biopsy.

\section{MANAGEMENT OF LUPUS NEPHRITIS}

Lupus nephritis is one of the best-studied SLE complications. The reasons are two-fold: 1 ) the morbidity and mortality are increased in SLE patients with renal involvement; 2) kidney biopsies, as opposed to serum autoantibodies, allow for direct visualization of active and chronic damage to tissues. It is thus no surprise that a histomorphological classification emerged in $1964^{3}$, based solely on light microscopy. This first attempt classified patients into 3 groups: lupus glomerulitis; active lupus glomerulonephritis and membranous lupus glomerulonephritis. Few changes were made until the 2004 joint World Health Organization and ISN/RPS (International Society of Nephrology / Renal Pathology Society) classification, which is still used to this date (Table 1). 


\section{Table 1}

Abbreviated Lupus Nephritis ISN/RPS classification

\begin{tabular}{l|l}
\multicolumn{1}{c||}{ Class } & \multicolumn{1}{c}{ Definition } \\
\hline Class I & Minimal mesangial lupus nephritis \\
\hline Class II & Mesangial proliferative lupus nephritis \\
\hline Class III & Focal lupus nephritis \\
\hline Class IV & Diffuse lupus nephritis \\
Class V & Lupus membranous nephropathy \\
Class VI & Advanced sclerosing lupus nephritis \\
\hline
\end{tabular}

Having a histopathologic classification is soothing for nephrologists, but its limitations must be recognized. Like the ACR and SLICC classification criteria, the ISN/RPS classification is imperfect - it catalogs kidney biopsy findings in 6 classes, not necessarily based on the underlying disease pathophysiology, the target for which immunomodulation is employed in LN. The best treatment for lupus nephritis is an eternal debate ${ }^{4}$, with several novel drug trials failing to prove superiority over conventional treatment. The explanation may lie in the failure to acknowledge that LN represents a myriad of concomitant pathophysiological mechanisms, with no "one size fits all" treatment. Arguably, to improve clinical outcomes, immunosuppressive treatment should be fine-tuned towards molecular signatures, instead of morphological findings. ${ }^{5}$

A kidney biopsy is nonetheless the best tool currently available in LN, and the ISN/RPS classification has been subject to frequent review since its first publication. The current classification has integrated the notion of activity and chronicity quantification indexes to LN class III and IV (before this, the terms A, A/C and C were used to grossly classify the findings as active and/or chronic LN) and the suppression of the class IV sub-classification as global or segmental (which was operator-dependent and had little clinical significance). ${ }^{6}$

For review and clarity, we will focus on and compartmentalize the management of $L N$ in 3 main sections:

i) Treating non-proliferative, non-membranous LN

ii) Treating proliferative $\mathrm{LN}$ - induction and maintenance

iii) Treating membranous $\mathrm{LN}$ - induction and maintenance

We will also be brushing lightly on adjunctive treatment, refractory disease and relapses.

\section{TREATING NON-PROLIFERATIVE, NON-MEMBRANOUS LN}

\section{Class I and II lupus nephritis}

In this subtype of $L N$, low levels of immune complexes are deposited within the mesangium; light microscopy may reveal absent proliferation (class I) or proliferation limited to the mesangium (class II). These patients have mild renal manifestations, usually presenting with hematuria and/or proteinuria. Patients with class I and II LN have an excellent renal prognosis and immunosuppression is generally not necessary unless required for extra-renal manifestations. Care should be directed towards blood pressure control (preferably through the blockade of the renin-angiotensin-aldosterone system), cardiovascular risk management and use of antimalarials such as hydroxychloroquine.

A notable exception is when nephrotic proteinuria is present, which may represent lupus podocytopathy, an entity that mimics minimal-change disease, with a comparable favorable response with a short steroid course. ${ }^{7}$ Electron microscopy may be useful when lupus podocytopathy is suspected since it can unmask podocyte injury, which is typically out of proportion to immunological activity. Some of those patients may have a more focal segmental glomerulosclerosis (FSGS) -like profile and may benefit from alternative immunosuppressive agents for steroid-resistant disease or as steroid sparing.

Class switching is frequent and it may need therapy change. A re-biopsy should be considered in this group of patients if they develop increased proteinuria and/or renal dysfunction.

\section{Class VI lupus nephritis}

This subclass represents irreversible scaring findings (i.e. $>90 \%$ of glomeruli are sclerosed and severe tubulointerstitial scaring), most likely because of prior persistent immunological activity. These patients have advanced fibrotic kidney disease, associated with progression towards ESRD. It is important to acknowledge that patients may have proteinuria, which in this situation is a sign of scaring and not of disease activity. Hence, immunosuppression is only indicated for treating extra-renal disease, and care should be in slowing down the progression of renal disease and preparation for initiation of renal replacement therapy.

\section{TREATING PROLIFERATIVE LN}

\section{Class III and IV lupus nephritis}

The histomorphological lesions that characterize class III and IV are similar. Those two classes are distinguished by the involvement of less or more than $50 \%$ of glomeruli (corresponding respectively to class III or class IV). Both classes share the same pathophysiological mechanisms of deposition of immune complexes in the subendothelial space of the glomerular capillaries, where they induce endothelial inflammation. This will lead to B-cell and plasma cell proliferation, causing intrarenal autoantibody production and local inflammation and proliferation. These changes result in a variable clinical picture - the most typical renal presentation is with acute kidney injury and active urinary sediment, but it can vary from otherwise asymptomatic hematoproteinuria to full-blown rapidly progressive renal failure.

Unless otherwise contraindicated, patients with proliferative LN should be referred for immunosuppression as soon as possible. As with many immunological diseases, immunosuppressive treatment in $L N$ is regarded as having two distinct consecutive phases:

i) Induction/initial therapy, where the goal is to control organ and life-threatening disease by aggressively reducing immunological activity. Most protocols last between 3 to 6 months; 
ii) Maintenance/subsequent therapy, where immunosuppression is less intensive. The aim is dual: to maintain clinical remission while having the lowest possible immunosuppressive burden and preventing relapses. Duration of maintenance therapy is a controversial topic, which is later explored.

Around $50-60 \%$ of patients achieve complete or partial remission during the first months of therapy (corresponding to induction therapy), but higher rates are achieved during the subsequent maintenance period. Those two periods are interlinked but, for the sake of clarity, the discussion is made separately.

\section{Induction}

\section{The current standard of care}

Active proliferative lupus nephritis can severely damage kidney function - up to $44 \%$ of patients with class III or IV LN progress to ESRD by 15 years. ${ }^{8}$ For this reason, immunological activity requires intensive immunosuppression. The currently recommended therapy for proliferative $L N$ involves a combination of high-dose corticosteroids with either mycophenolate mofetil (MMF) or cyclophosphamide (CYC). These recommendations emerged after many years of landmark trials, with the first publication of LN treatment leading back to 1964 by Pollak and his colleagues ${ }^{3}$, where they provided the first evidence for the utility of high-dose steroids. However, survival was poor, an aspect which only improved with the introduction of combined immunosuppressive protocols. In 1986, Austin et al ${ }^{9}$ published the National Institutes of Health $(\mathrm{NIH})$ protocol comparing oral prednisone monotherapy versus four combination protocols (oral azathioprine and/or oral CYC; intravenous CYC; in all arms patients also received steroids) where the intravenous CYC regimen (six-monthly $0.5-1 \mathrm{~g} / \mathrm{m}^{2}$ infusions) established itself as the one achieving the biggest reduction in ESRD risk; prednisone alone was the worse regimen on this metric. The oral CYC regimen, besides having worse outcome than IV CYC, is associated with higher cumulative dose and higher risk of toxicity.

Because avoidance of ESRD was a somewhat unambitious therapy-goal, the focus was now changing towards achieving clinical remission. It should be stated that the definitions of complete and partial remission are not consensual, but typically include a combination of improved glomerular filtration rate and proteinuria and inactive urinary sediment. Because of the lack of a clear-cut definition, many trials have used different targets, achieving results that are sometimes not comparable and leading to confusion. ${ }^{10}$ Besides remission rates, there were increasing concerns over adverse events due to excessive immunosuppressive exposure, namely CYC-induced ovarian failure. The 2002 EuroLupus trial ${ }^{11}$ compared standard-dose IV CYC (like the NIH protocol) with a low-dose IV CYC regimen (six biweekly $0.5 \mathrm{~g}$ infusions), in both cases followed by azathioprine (AZA) maintenance. Cumulative $\mathrm{CYC}$ dose in the two arms was $8.5 \pm 1.9 \mathrm{~g}$ vs $3 \mathrm{~g}$, respectively. The lower-dose EuroLupus regimen achieved similar remission rates and renal flares remained similar after 10 years of follow-up. ${ }^{12}$ While there was a lower frequency of serious infectious complications in the low-dose group, it did not reach statistical significance. One limitation is that the EuroLupus population consisted mainly of Caucasian patients, which holds back the adoption of a low-dose CYC regimen in North America, where there is a more varied ethnic population, who tend to have more severe and refractory nephritis. Additionally, only $22 \%$ of patients had a serum creatinine $>1.3 \mathrm{mg} / \mathrm{dl}$, raising questions about the efficacy of lower-dose CYC regimen in more serious disease.

MMF emerged as a possible induction therapy option after the publication of a trial where 42 Hong-Kong patients treated with oral MMF or IV CYC as induction and followed by azathioprine and prednisolone maintenance. Both arms had similar remission rates, but higher relapse rates during the 3-year follow-up were observed in the MMF arm. ${ }^{13}$ In the 2009 landmark Aspreva Lupus Management Trial $(A L M S)^{14}, 370$ patients were randomized to either oral MMF or standard dose IV CYC, including 32 patients with eGFR $<30 \mathrm{ml} / \mathrm{min} / \mathrm{m}^{2}$. After 24 weeks, patients who responded were randomized to either maintenance MMF or AZA. The study found similar renal outcomes with both induction regimens. However, the more varied ethnic population in this study showed that Black and Hispanic patients had a higher remission rate under MMF induction, with similar frequency of adverse events. Although there are no studies with mycophenolic acid, it is regarded as equivalent to MMF.

An interesting posthoc analysis of the ALMS cohort investigated whether MMF or CYC had better results in patients with severely decreased renal function (GFR less than $30 \mathrm{~mL} / \mathrm{min}$ ). Response rates were similar ( $20 \%$ for the MMF group vs. $17 \%$ for the IV CYC group). Both groups had higher serious adverse events rate when compared with the overall study population (52\% vs. $25 \%) .{ }^{15}$

Corticosteroids are a standard component of all treatment regimens in lupus nephritis, usually with a high IV methylprednisolone start dose (1-3 pulses of $500-1000 \mathrm{mg}$ ), followed by oral prednisolone (0.5-1 $\mathrm{mg} / \mathrm{kg} /$ day), which is tapered over 6 months to a low daily dose. While the utility of corticosteroids in LN is unquestionable, the associated adverse events are frequent and can be serious. Their image-altering effects are a major concern for patients, being a driving force for poor adherence. Despite this and of years of use, there is little evidence on the ideal dose, tapering schedule and duration of therapy - in fact, decisions on dose and duration of steroid treatment appear to be more dependent on the physician preference than on patients' clinical response. ${ }^{16}$ But there has been an effort towards steroid-sparing regimens. In severe SLE, doses of methylprednisolone of $500 \mathrm{mg}$ a day were found to be comparable to $1000 \mathrm{mg}$ a day. ${ }^{17}$ In LN, a small-randomized control trial (RCT) compared standard $(1 \mathrm{mg} / \mathrm{kg})$ and reduced $(0.5 \mathrm{mg} / \mathrm{kg})$ prednisolone dosage, and found similar short-term outcomes with fewer infectious events. ${ }^{18}$

Besides dosage minimizing, another approach is to substitute them with other drugs with a more benign side effects profile. This has led to one of the most innovative attempts at steroid-sparing, proposed by the Hammersmith group, who used rituximab for minimizing steroid exposure (which we explore in the B-cell therapy section below). ${ }^{19} \mathrm{~A}$ recent phase 2 RCT used a combination of a new calcineurin inhibitor (CNI) molecule (voclosporin), MMF and a very rapid steroid taper, from a maximum prednisolone start dose of $25 \mathrm{mg}$, down to $5 \mathrm{mg}$ by 8 weeks, a dosage usually attained by week 24 in most trials; the trial met their remission end-points by week $48 .^{20}$

These investigations will hopefully lead the way for other trials specifically directed towards the optimization of steroids dosage and duration. 


\section{Second-line therapies}

\section{a) Azathioprine (AZA)}

In the already mentioned classic NIH trial, AZA was found to be less effective than IV CYC in inducing remission. In a 2006 Dutch RCT comparing AZA against CYC, the former group was found to have a similar doubling of serum creatinine, albeit with more common relapses and herpes zoster infections during follow-up. ${ }^{21}$ Based on these findings, AZA is currently not considered first-line therapy for $L N$.

\section{b) B-cell therapies}

Because $B$ cells play a critical role in the pathogenesis of SLE, great hope was put into $B$ cell-depleting drugs for the treatment of lupus nephritis. Rituximab depletes auto reactive $B$ cells and its use has been studied in LN. While many successful case reports in LN-resistant disease are available, RCTs have been unable to show benefit in using rituximab in LN. The 144-patients LUNAR trial ${ }^{22}$ evaluated rituximab as add-on therapy to MMF and corticosteroids - it did result in reduced anti-dsDNA and improved C3 and C4, but this did not translate into improved clinical outcomes after 12 months. Ocrelizumab, a second-generation monoclonal anti-CD20 antibody, also had a disappointing outcome as an add-on drug (to steroids and either MMF/CYC) in the BELONG trial ${ }^{23}$, which was stopped early due to a higher rate of serious infections in the ocrelizumab arm, especially in the MMF arm. Taken together, these trials show that anti-B-cell therapies can reduce inflammation but have failed to achieve clinical outcome improvement in LN. Many putative explanations have emerged, including the limited number of patients, short follow-up, overtreatment of mild disease, and the fact that it was used as add-on therapy (in contrast with the trials that led to CYC and MMF to be used in proliferative $L N$ ).

While the evidence does not favor the use of B-cell therapies as an add-on in induction therapy, they may be useful in resistant disease. They may also play a part as a steroid-sparing agent. The investigator-led RITUXILUP trial was aimed at comparing a standard MMF and steroid arm against an MMF-based regimen in combination with rituximab, devoid of steroids. The pilot study ${ }^{19}$ suggested that rituximab could indeed enable oral steroid dose reduction. Unfortunately, the RITUXILUP trial was terminated early due to slow recruitment and withdrawal of funding.

An indirect way of reducing B-cell activity is through the inhibition of B-cell-activating factor (BAFF, also known as BLyS). Belimumab is a monoclonal antibody that neutralizes BAFF, thereby inhibiting B-cell survival and differentiation. Following the landmark trials BLISS $52^{24}$ and BLISS $76^{25}$ - which excluded patients with central nervous system or renal involvement - belimumab is now the only therapy to be approved or licensed for use in SLE in over 50 years. However, evidence towards routine use in NL is still lacking; the BLISS-LN trial will hopefully fill this gap in knowledge (ClinicalTrials.gov Identifier NCT01639339). Following B-cell depletion with rituximab, a BAFF level increase is seen, which some suspect may explain the production of auto reactive $B$ cells and resistance to rituximab. This is the rationale for a sequential rituximab and belimumab therapy, the first as induction therapy and the second as maintenance (vs. placebo), which is being studied in patients with LN in the CALIBRATE study (ClinicalTrials.gov Identifier NCT02260934). Although theoretically attractive, the preliminary results were less encouraging, with similar renal outcomes at 48 weeks. ${ }^{26}$
None of the above drugs can deplete antibody-producing plasma cells. This can be achieved using proteasome inhibitors, such as bortezomib. The use of such agents may sometime find its place in treating LN but should currently be considered only experimental.

\section{c) Calcineurin inhibitors (CNIs) and multitarget strategy}

The use of CNIs in combination with steroids in LN has been perceived as an effective and potentially less toxic option. They decrease proteinuria through both immunosuppressive properties and the stabilization of podocyte cytoskeleton. A recent meta-analysis showed that CNIs or their combination with MMF was comparable to IV CYC in inducing remission in proliferative LN ${ }^{27}$ It should be noted that those results are driven mainly by trials in Asian patients. CNIs have no negative effect on fertility and pregnancy, unlike CYC and MMF. However, CNIs do have shortcomings: relapse rates have been reported to be higher after their suspension ${ }^{28}$; they have a narrow therapeutic window and there are concerns that exposure to CNIs may induce nephrotoxicity (both acute and chronic).

An interesting approach is to use CNIs as adjunctive to steroids and MMF, to target multiple immune mechanisms - a strategy used in kidney transplantation and that has come to be known as multitarget. This could allow for lower drug dose (and hopefully less toxicity), while maintaining remission rates. In a Chinese population ${ }^{29}$, an approach combining steroids, MMF and tacrolimus achieved better outcomes than a standard regimen of steroids and IV CYC, with more treatment withdrawals in the multitarget arm. More recently, very high rates of remission were obtained in the AURA-LV phase 2 trial, which used a similar multitarget approach with a new CNI drug (voclosporin) in a more ethnic-diverse population. ${ }^{20}$

Despite those promising results, one should note that remission criteria are largely based on proteinuria reduction. Given the podocyte-stabilization properties of CNIs, remission rates should be analyzed with caution. Repeat renal biopsies will prove instrumental in distinguishing proteinuria reduction caused by CNIs' antiproteinuric effects from true renal remission - additionally, work will have to be done to establish whether the distinction is clinically relevant in regards of long-term prognosis.

Although the term multitarget is usually used when referring to CNI-including regimens, the same approach was used with other drugs, such as the previously mentioned LUNAR trial. A recent meta-analysis of trials comparing double (i.e. classic) versus triple-immunosuppressive regimens was published - of the 11 included trials, 6 of them included biologics ( 3 rituximab, 2 abatacept, 1 ocrelizumab). ${ }^{30}$ They found higher remission rates in the multitarget arm without biologics, while the multitarget arm with biologics improved outcomes only in refractory severe LN; safety analysis showed similar results in all arms, possibly because drug doses used in most multitarget trials is lower than routine doses. The remission rates of triple-drug regimens are encouraging, but the long-term benefit is still unknown. It will also bring new challenges, such as the early identification of which patients benefit from classic or triple regimens (with or without biologics) and for how long.

The current research pipeline contemplates additional drugs which may find their place in the treatment of proliferative LN. Some of them include approaches involving co-stimulatory blockade and 
anti-cytokine therapies, but are not expected to be available in the near future for the treatment of $L N$, and are outside the scope of this review. Until then, regimens with $\mathrm{CYC}$ and MMF are the recommended options, with the potential added benefit of the latter for African-American and Hispanic patients.

\section{Maintenance}

\section{The current standard of care}

Maintenance therapy consists of less intensive immunomodulation, with the goal of consolidating clinical remission and preventing disease relapses. Lack of maintenance therapy is associated with worse clinical outcomes. Since SLE's natural history consists of periods of flares and remission, enough immunosuppression must be given to prevent the former, even after remission is obtained. However, prolonged exposure to immunosuppressive drugs is associated with toxicity. The conundrum is to attain just the right dose, lasting only until necessary.

\section{Treatment options}

MMF and AZA are the two drugs currently recommended for maintenance phase in proliferative LN. Of note, these oral drugs replaced the use of quarterly maintenance IV CYC after a 59-patient trial showed they were both safer and more effective in maintaining remission. ${ }^{31}$

These recommendations were further confirmed following two pivotal trials - the MAINTAIN trial $^{32}$ and the ALMS maintenance phase trial $^{33}$ :

i) The MAINTAIN nephritis trial selected patients who been subjected to a EuroLupus low-dose IV CYC induction to be randomized to either MMF (target dose: $2 \mathrm{~g} /$ day) or AZA (target dose: $2 \mathrm{mg} / \mathrm{kg} /$ day) - a population of 105 mostly Caucasian patients were followed-up for 48 months. No significant differences were found in this population, although there was a tendency for fewer relapses in the MMF group and more hematologic cytopenias in the AZA group.

ii) The ALMS trial induction phase compared MMF to standard dose IV CYC. Patients who responded to induction therapy were invited to participate in the maintenance phase of the ALMS trial, resulting in 227 patients randomized to maintenance therapy with MMF or AZA (target dose: $2 \mathrm{~g} /$ day and $2 \mathrm{mg} / \mathrm{kg} /$ day, respectively) during a follow-up of 36 months. This population was more ethnically diverse than the MAINTAIN trial. Treatment failure (defined as a composite outcome of death, ESRD, doubling of serum creatinine, renal flare and rescue treatment) was more frequent in the AZA arm, resulting in MMF to be considered a superior option, regardless of race.

While MMF and AZA are the recommended options, calcineurin inhibitors have also been proposed as an option for maintenance in $\mathrm{LN}$, but the evidence is weak. Cyclosporine was compared to AZA in a 2006 trial, with 75 patients reported to have similar renal flare rate after 4 years of follow-up; no evidence of CNI-nephropathy was seen in the 29 patients who had repeat biopsy at the 2-year follow-up mark. ${ }^{34}$ A subsequent 70-patient Chinese cohort compared maintenance with AZA or tacrolimus. ${ }^{35}$ Although flare rates were similar, results should be interpreted with caution since published follow-up was only 6 months.
Following the encouraging results from the above-mentioned multitarget (tacrolimus, MMF and prednisolone) induction trial, patients who responded to either multitarget or CYC therapy at 24 weeks were invited for a maintenance trial with multitarget or azathioprine therapy, respectively. After 18 months of maintenance therapy, the rate of relapses was similar, but the number of adverse events was higher in the AZA group (44\% vs. $16 \%) .{ }^{36}$ While multitarget therapy may become an option for maintenance therapy, some answers are still needed: are the results generalizable beyond Asian populations? Will CNI-induced nephrotoxicity be an issue? Will patients maintain remission when/if CNIs are weaned off?

\section{Treatment duration}

Clinical improvement in proliferative LN continues beyond induction, making the need for maintenance therapy in LN indisputable. However, its ideal duration remains controversial. KDIGO guidelines suggest consideration of slow taper in patients who have achieved complete remission for at least a year but make no consideration on the total length of therapy. ${ }^{37}$ The complete withdraw of immunosuppressive drugs is a controversial topic. Due to concerns over immunosuppression overexposure and its complications, therapy cessation will put patients at risk of having a disease flare. ${ }^{38}$

The consensus is that therapy withdrawal should only be attempted in selected cases, when remission is unequivocal and under strict surveillance. In an Italian cohort, therapy withdrawal was attempted in 73 patients, 21 of which flared during therapy weaning. Of the 52 patients who completed treatment withdrawal, lasting remission was observed in $61.5 \%$ of patients, while $38.5 \%$ developed new flares after a median of 37 months. Longer duration of treatment before the interruption of therapy and the use of antimalarial agents were associated with relapse-free withdrawal. ${ }^{39}$

In patients where remission is difficult to attain or who have relapsed in the past, further caution is needed and interrupting maintenance treatment is discouraged. Some high-risk patients may need life-long immunosuppressive treatment. While this may seem a gruesome sentence, it is important to put things in perspective: in the event of relapse due to premature weaning, the patient is at risk of ESRD and dialysis, where kidney transplantation remains the best option - if that happens, the patient will face lifelong immunosuppressive treatment anyway.

Between those two extremes lie most patients with LN. The current lack of consensus on biomarkers of remission makes therapy withdrawal a challenge for clinicians. Further considerations about maintenance and relapse risk are made in a later section.

\section{TREATING MEMBRANOUS LN}

\section{Class V lupus nephritis}

Membranous LN is a secondary cause of membranous glomerulopathy. It accounts for $10-20 \%$ of lupus nephritis cases and may co-exist with proliferative class III or IV LN. When this is the case, the prognosis is similar to proliferative $L N$, and management is usually directed towards the latter. Within this section, we will consider isolated membranous LN. 
Class V LN occurs following the deposition of immune complexes in the glomerular sub-epithelial compartment, which entices complement activation and podocyte injury; proliferation is usually mild or absent. For this reason, patients typically manifest with proteinuria, frequently in the nephrotic-range, with little renal function change. Renal prognosis is usually better than proliferative lupus and can sometimes be managed without immunosuppression. All patients should be initiated on therapy directed towards proteinuria control, including strict hypertension management. Renin-angiotensin-aldosterone system (RAAS) blockers are the mainstay; dual RAAS-blockage and nondihydropyridine calcium-channel blockers may also be considered.

Patients with subnephrotic proteinuria in the context of membranous lupus nephritis have an excellent renal prognosis, and frequently do not require additional immunomodulatory treatment. However, the consensus is that spontaneous remission is infrequent in patients with nephrotic-range proteinuria or decline in renal function. Additionally, higher proteinuria is associated with increased thrombotic risk, which may require prophylactic anticoagulation. Patients with nephrotic-range proteinuria or deteriorated renal function will evolve

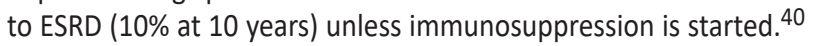

High quality randomized controlled trials in pure membranous $L N$ are lacking. Since membranous $L N$ represents a smaller fraction of all LN patients, less evidence is available. Therefore, most information is gained from small trials and through sub-analysis of large $L N$ trials and from studies of primary membranous nephropathy. ${ }^{40}$ Further complicating matters, resolution of proteinuria is time-dependent, since it requires immune deposits reabsorption and resolution of glomerular basement membrane abnormalities. Because those typically lag behind immunological activity cessation, proteinuria may persist for longer than expected, and it is important to distinguish it from non-response. Similar to proliferative LN, therapy is divided into induction and maintenance phases.

\section{Induction}

As for proliferative $L N$, the evidence for prednisolone dose is weak, but the established recommended dose is $0.5-1 \mathrm{mg} / \mathrm{kg} /$ day. A small RCT included 42 patients with membranous $L N$ and randomized them to three treatment arms: i) steroids alone; ii) steroids with cyclophosphamide; iii) steroids with cyclosporine. At one-year follow-up, remission rates were lower in the steroid monotherapy group (27\%,60\% and $83 \%$, respectively). Patients randomized to cyclosporine achieved quicker remission but were noted to have more relapses. ${ }^{41}$ This is clear evidence of proteinuria reduction through non-immune mechanisms. ${ }^{42}$ Clinicians need to be aware that CNI therapy duration may need to be more prolonged than with standard regimens.

The pooled analysis of 84 patients with pure membranous LN included in two large LN trials comparing induction with MMF vs. CYC showed no difference in proteinuria reduction, remission rate or side effects was found, but the follow-up was limited to 24 weeks. ${ }^{43}$

Treatment with azathioprine is also considered to be effective and safe although with no comparative trials. ${ }^{44}$

The above-mentioned multitarget induction trial ${ }^{29}$ also included 69 patients with isolated membranous $L N$; higher remission rates were also observed in the multitarget group vs. patients under CYC. Intriguingly, in the phase 2 trial AURA-IV, which also used a similar multitarget regimen (albeit with voclosporin) with higher than expected remission rates, results were driven by patients without isolated class $\mathrm{V} \mathrm{NL}$ patients, a finding that will be interesting to confirm in the upcoming phase 3 trial. $^{20}$

B-cell depleting monoclonal antibody therapy is also an option. There is some evidence of the efficacy of rituximab for class V LN, but it remains not well established. ${ }^{19,45}$

\section{Maintenance}

Current evidence for maintenance phase in pure membranous LN is even more unsatisfying. Most guidelines and expert opinion suggest either MMF or AZA. A recent meta-analysis did not find differences in efficacy and safety between TAC, MMF, AZA or CYC. ${ }^{46}$

As it is for proliferative LN, the optimal duration of treatment is unknown. Recommendations are to maintain immunosuppressive treatment during 2-4 years before tapering therapy.

In summary, guidance for treating membranous lupus nephritis is lacking. The lower number of patients with class V LN means we are unlikely to see a trial dedicated to this group of patients. However, we will probably continue to gain evidence from subgroup analysis of larger trials. Until then, multiple treatment options appear to be equally effective, with current expert-opinion generally favoring the use of MMF for both induction and maintenance therapy.

\section{REFRACTORY AND RELAPSING LUPUS NEPHRITIS}

SLE and its manifestations are characterized by periods of remission and activity. As explored in the previous sections, long-term immunosuppression is required to obtain a lasting remission. Two challenging scenarios may occur: not being able to obtain remission with a first-line therapy or, following clinical remission, LN disease re-emerges. Both occurrences are relevant and physicians who care for $L N$ patients should be prepared to manage them.

\section{Refractory LN}

High-quality evidence regarding the treatment of refractory $L N$ is lacking, starting with the absence of a clear-cut definition - for instance, EULAR guidelines consider 3 kinds of refractory LN: i) those who fail to improve after 3-4 months of therapy; ii) those who do not achieve partial remission after 6-12 months; iii) those who do not reach complete renal remission after 2 years of treatment. ${ }^{47}$ This classification is unsatisfactory because it tries to include clinical remission (which is already hard to agree upon) with the timing at which it is expected to occur - which depends on a myriad of factors and is hard to predict.

Management in such cases is difficult, and most often based on expert opinion. It is important to try to establish the cause of non-response. Non-adherence is a well-recognized cause of treatment failure-some treatments have significant secondary effects, which 
may be the reason for non-adherence. One way of assessing adherence is through drug monitoring. While MMF has proved particularly challenging to monitor, both CNIs and hydroxychloroquine can be. By identifying non-adherent patients, one can also modify our approach to support them appropriately.

Repeating renal biopsy may also contribute to the management of refractory cases, since some patients may have since the previous biopsy developed extensive fibrosis, where more immunosuppression would be detrimental. On the contrary, if immunomodulation is thought to be required, it is generally recommended to switch to another conventional induction therapy, i.e., if the patient was initially treated with $\mathrm{CYC}$, it is suggested to attempt remission with MMF, and vice-versa. Other options are to add or change to anti-CD20 therapy, a multitarget approach with CNI (in particular if there is significant proteinuria) or treatment with intravenous immunoglobulin (IVIg) or plasma exchange (PLEX) - but the evidence is generally of low quality. There are a substantial number of successful case reports of rituximab use in refractory disease, in contrast with its application in induction therapy. ${ }^{48}$ For this reason, rituximab was being evaluated in a randomized clinical trial in refractory LN (ClinicalTrials.gov Identifier NCT01673295) but was terminated prematurely due to lack of recruitment, which leads to the question whether refractory $L N$ is as frequent as once thought.

In summary, management optimization is key in patients deemed to have refractory LN. This may include non-adherence detection, adjunctive treatment such as ACE inhibitors or ARBs and hydroxychloroquine, repeat kidney biopsy and the switch to other immunosuppressive treatment. While many options are available for treating patients who do not respond to standard induction regimens, experts tend to agree on anti-CD20 therapy as the best option. However, the definition of refractory $L N$ seems to have been overstepped, resulting in lack of trial comparability. While a consensus definition is not achieved, recommendations will remain based on expert-opinion.

\section{Relapsing LN}

\section{Relapse risk factors and identification}

Maintenance therapy in LN is designed to consolidate remission and prevent relapses. But even when long-term complete remission is obtained, there is a risk of LN relapse, which is estimated to happen in $50 \%$ of patients within the first five years of therapy. How can we predict which patients will relapse? Sensitive and reliable predictors are lacking, but some risk factors have been identified, the most predictive one being whether the patient attained complete remission or not. Hard renal outcomes were evaluated in a follow-up analysis of the ALMS and EuroLupus trials, where both found that proteinuria at 12 months was the best predictor of long-term renal function. ${ }^{49} \mathrm{It}$ makes sense that the patients whose disease remits quicker will also be the ones with lower relapse risk. This should be kept in consideration when evaluating trials which include $\mathrm{CNIs}$, since they reduce proteinuria through non-immunosuppressive pathways, hampering the use of clinical remission as currently defined - and perhaps explaining a tendency for higher relapse in protocols including CNIs. Other predictors of relapse include high activity index in kidney biopsy, male gender, younger age, hypertension and delay in initiation of treatment. ${ }^{50}$
While relapse is sometimes hard to predict, careful monitoring of high-risk patients is arguably the best way to detect and treat relapses in early stages. Urinary and serum biomarkers may in the future play a significant role in identifying such patients. ${ }^{51}$ For now, assessment of clinical SLE activity, autoimmune serology, renal function and automated urinary analysis are the mainstay. Other strategies can be put in place:

i) Drug monitoring can prove instrumental in achieving appropriate drug levels and in unmasking non-adherence.

ii) Urinary sediment: Although valuable, automated urinary analysis cannot distinguish isomorphic from dysmorphic erythrocytes, has trouble in differentiating casts types and cannot integrate the urinary findings with the clinical picture, all of which are essential for nephrologists evaluating a patient with suspected/ known glomerular disease. In SLE patients, urinary sediment can be an important tool and can suggest the histological LN class. ${ }^{52}$ Urinalysis and urinary sediment findings can also help predict relapses in $\mathrm{LN}^{53}$ and in ANCA-vasculitis. ${ }^{54}$ Conversely, worsening renal function combined with the absence of inflammatory changes in the urinary sediment can indicate non-immune mechanisms at play, which will not benefit from immunosuppression. Urinary sediment analysis is nonetheless time-consuming, observer-dependent and not widely available.

iii) Repeat kidney biopsies: Our current definition of remission relies on renal function, urinary sediment and proteinuria. There is mounting evidence that this is insufficient, and may even be one of the reasons why so many trials have failed in LN. There is a case to be made in favor of integrating histology in the definition of renal remission: in an Argentinian cohort, 69 patients were re-biopsied after 6 months of induction therapy - one-third of those with complete clinical response had persistently high histologic activity; $62 \%$ of patients who had complete histologic remission were still clinically active. ${ }^{55}$ Biopsy findings were discordant from clinical findings, which shows there is room for improvement with our current biomarkers; importantly, patients who maintained higher histologic activity were the ones with worse long-term renal outcomes.

Expert opinion guideline recommendations suggest immunosuppression weaning after 36 months of treatment (including at least 12 months of complete remission). Compelling data came from a trial that put those recommendations to test: patients who fulfilled the two conditions performed a kidney biopsy; 36 patients were follow-up during 24 months. Despite no clinical activity at the time of tapper, biopsy findings were varied: 20 patients had complete histologic remission; 9 had vestigial activity (activity index 1 or 2); 7 had an activity index between 3 and 5 . Histologic findings at this time were not used for clinical management and all patients underwent the recommended maintenance drug taper and discontinuation. During the 24-month follow-up, LN flare occurred in 11 patients (30.6\%). All but 1 flare occurred in patients where there was remaining histologic activity. On multivariable analysis, two risk factors emerged: the renal biopsy activity index at the time of taper and SLE duration. ${ }^{56}$ The elephant in the room is whether continued maintenance would have avoided LN relapse.

Protocol biopsies were recently used in a $\mathrm{LN}$ cohort to decide whether to discontinue maintenance therapy. ${ }^{57}$ Patients were only 
weaned off therapy if no histologic activity was found; a low flare rate and low rate of renal-biopsy related complications suggest this strategy may be appropriate in this scenario.

Repeat biopsies have the potential to help interpret our current incomplete biomarkers and can identify ongoing histological activity, increased interstitial fibrosis or other non immune-mediated lesions, and will probably be used increasingly in LN. In the future, the definition of complete remission might evolve to include the notion of complete histologic remission.

\section{Relapse treatment}

The treatment of $L N$ relapses depends upon the initial immunosuppression and the current medication and disease severity. If the patient has a mild disease (i.e. stable renal function but increased immunological activity, increased proteinuria and/or inflammatory urinary sediment changes), restarting or increasing steroids and MMF/azathioprine may be enough to counteract the increase in disease activity. However, for patients with more aggressive disease course, immunosuppressive therapy needs to be significantly increased. The usual choice of therapy is to repeat the induction treatment that allowed for clinical remission the first time around. Nevertheless, the risk of toxicity is higher due to previous drug exposure. To avoid cumulative CYC toxicity, some experts prefer MMF in relapsing disease regardless of prior induction regimen. On the other hand, if the patient suffers a severe relapse while still under MMF maintenance therapy, a CYC regimen is the preferred choice. For patients who relapse frequently, B-cell therapy may be beneficial.

\section{ADJUNCTIVE TREATMENT AND AVOIDING IATROGENESIS}

Judicious use of immunosuppressive drugs to avoid iatrogenesis during the treatment of lupus nephritis is essential and is the reason for the development of steroid-sparing protocols, such as the RITUXILUP and multitarget approaches. Additional adjunctive therapy can help manage $\mathrm{LN}$ activity and protective drugs are frequently necessary to avoid iatrogenesis.

Antimalarials such as hydroxychloroquine (HCQ) are safe drugs that are recommended for all SLE patients since they decrease thrombotic events, ESRD and mortality. (58-60) Because HCQ may rarely cause retinal toxicity, ophthalmological monitoring is required. In patients with $\mathrm{LN}$, antimalarials improve remission rates, whilst their withdrawal increases the risk of relapse. ${ }^{61,62}$ Mortality is lower in SLE patients who keep taking HCQ despite requiring dialysis. ${ }^{63}$

Since immunosuppressive drugs are associated with significant adverse events, special care should be given to prevent infection (pneumocystis jirovecci pneumonia in particular), and to prevent steroid-induced osteoporosis, gastrointestinal bleeding, dyslipidemia and hypertension. Although of fundamental importance, this topic is somewhat beyond the scope of this review.

Since most patients with $\mathrm{LN}$ are young, attention should be given to fertility preservation and pregnancy. CYC is known to be gonadotoxic and potentially causing infertility in both male and female patients, something which may be preventable if care is taken in a timely fashion. Many of the frequently used drugs in LN are teratogenic (ACE inhibitors, ARBs, MMF, CYC, warfarin), whilst safe options are also available (steroids, AZA, CNIs, HCQ). It is crucial to discuss pregnancy wish with all patients in childbearing age (including male patients under MMF), to adequately prepare and prevent undesirable maternal and fetal outcomes.

\section{CONCLUSION}

Lupus nephritis is a common and serious complication of SLE. While in some clinical scenarios, diagnosis and treatment decisions are straightforward and evidence-based, this is not true for many others. This reflects our current lack of both solid disease activity definitions and of specific and non-invasive biomarkers. Until these are developed, clinical judgment and kidney biopsy will remain the strongest weapons for nephrologists caring for lupus nephritis patients. Many drugs and strategies are currently in the research pipeline, hopefully leading the way for targeted individualized therapies.

Disclosure of potential conflicts of interest: none declared

\section{References}

1. Kaul A, Gordon C, Crow MK, et al. Systemic lupus erythematosus. Nat Rev Dis Prim. 2016;2(1):16039.

2. Danila MI, Pons-Estel GJ, Zhang J, et al. Renal damage is the most important predictor of mortality within the damage index: data from LUMINA LXIV, a multiethnic US cohort. Rheumatology. 2008; $48(5): 542-545$.

3. Pollak V, Pirani C, Schwartz F. The natural history of the renal manifestations of systemic lupus erythematosus. J Lab Clin Med. 1964;63(4):537-550.

4. Bomback AS. An update on therapies for proliferative lupus nephritis: How certain can we be about the evidence? Am J Kidney Dis. 2018;72(5):758-760.

5. Yu F, Haas M, Glassock R, Zhao M-H. Redefining lupus nephritis: Clinical implications of pathophysiologic subtypes. Nat Rev Nephrol. 2017; 13(8):483-495.

6. Bajema IM, Wilhelmus S, Alpers CE, et al. Revision of the International Society of Nephrology/ Renal Pathology Society classification for lupus nephritis: Clarification of definitions, and modified National Institutes of Health activity and chronicity indices. Kidney Int. 2018;93(4):789-796.

7. Chen D, Hu W. Lupus podocytopathy: A distinct entity of lupus nephritis. J Nephrol. 2018;31(5):629-634 .

8. Sexton DJ, Reule S, Solid C, et al. ESRD From lupus nephritis in the United States, 1995-2010. Clin J Am Soc Nephrol. 2015;10(2):251-259.

9. Austin HA, Klippel JH, Balow JE, et al. Therapy of lupus nephritis. N Engl J Med. 1986;314(10):614-619.

10. Corapi KM, Dooley MA, Pendergraft WF. Comparison and evaluation of lupus nephritis response criteria in lupus activity indices and clinical trials. Arthritis Res Ther. 2015;17(1):110.

11. Houssiau FA, Vasconcelos C, D'Cruz D, et al. Immunosuppressive therapy in lupus nephritis: The Euro-Lupus Nephritis Trial, a randomized trial of low-dose versus high-dose intravenous cyclophosphamide. Arthritis Rheum. 2002;46(8):2121-2131.

12. Houssiau FA, Vasconcelos C, D'Cruz D, et al. The 10-year follow-up data of the Euro-Lupus Nephritis Trial comparing low-dose and high-dose intravenous cyclophosphamide. Ann Rheum Dis. 2010;69(01):61-64.

13. Chan TM, Li FK, Tang CSO, et al. Efficacy of mycophenolate mofetil in patients with diffuse proliferative lupus nephritis. N Engl J Med. 2000;343(16):1156-1162.

14. Appel GB, Contreras G, Dooley MA, et al. Mycophenolate Mofetil versus cyclophosphamide for induction treatment of lupus nephritis. J Am Soc Nephrol. 2009;20(5):1103-1112.

15. Walsh M, Solomons N, Lisk L, Jayne DRW. Mycophenolate mofetil or intravenous cyclophosphamide for lupus nephritis with poor kidney function: A subgroup analysis of the aspreva lupus management study. Am J Kidney Dis. 2013;61(5):710-715.

16. Walsh $M$, Jayne $D$, Moist $L$, et al. Practice pattern variation in oral glucocorticoid therapy after the induction of response in proliferative lupus nephritis. Lupus. 2010;19(5):628-633.

17. Badsha H, Edwards CJ. Intravenous pulses of methylprednisolone for systemic lupus erythematosus. Semin Arthritis Rheum. 2003;32(6):370-377.

18. Zeher M, Doria A, Lan J, et al. Efficacy and safety of enteric-coated mycophenolate sodium in combination with two glucocorticoid regimens for the treatment of active lupus nephritis. Lupus. 2011;20(14):1484-1493.

19. Condon MB, Ashby D, Pepper RJ, et al. Prospective observational single-centre cohort study to evaluate the effectiveness of treating lupus nephritis with rituximab and mycophenolate mofetil but no oral steroids. Ann Rheum Dis. 2013;72(8):1280-1286. 
20. Rovin BH, Solomons N, Pendergraft WF, et al. A randomized, controlled double-blind study comparing the efficacy and safety of dose-ranging voclosporin with placebo in achieving remission in patients with active lupus nephritis. Kidney Int. 2019;95(1):219-231.

21. Grootscholten C, Ligtenberg G, Hagen EC, et al. Azathioprine/methylprednisolone versus cyclophosphamide in proliferative lupus nephritis. A randomized controlled trial. Kidney Int. 2006:70(4):732-742.

22. Rovin $B H$, Furie $R$, Latinis $K$, et al. Efficacy and safety of rituximab in patients with active proliferative lupus nephritis: The lupus nephritis assessment with rituximab study. Arthritis Rheum. 2012;64(4):1215-1226.

23. Mysler EF, Spindler AJ, Guzman R, et al. Efficacy and safety of ocrelizumab in active proliferative lupus nephritis: Results from a randomized, double-blind, phase III study. Arthritis Rheum. 2013;65(9):2368-2379.

24. Navarra SV, Guzmán RM, Gallacher AE, et al. Efficacy and safety of belimumab in patients with active systemic lupus erythematosus: A randomised, placebo-controlled, phase 3 trial. Lancet. 2011;377(9767):721-731.

25. Furie $\mathrm{R}$, Petri $\mathrm{M}$, Zamani $\mathrm{O}$, et al. A phase III, randomized, placebo-controlled study of belimumab, a monoclonal antibody that inhibits B lymphocyte stimulator, in patients with systemic lupus erythematosus. Arthritis Rheum. 2011;63(12):3918-3930

26. Dall'Era M, Aranow C, Byron M, et al. Phase 2 Trial of Induction Therapy with Anti-CD20 (Rituximab) Followed By Maintenance Therapy with Anti-BAFF (Belimumab) in Patients with Active Lupus Nephritis. 2018 ACR / ARHP Annu Meet USA Chicago IL, 19-24 Oct 2018.

27. Palmer SC, Tunnicliffe DJ, Singh-Grewal D, et al. Induction and maintenance immunosuppression treatment of proliferative lupus nephritis: A network meta-analysis of randomized trials. Am J Kidney Dis. 2017;70(3):324-336.

28. Mok CC, Ying KY, Yim CW, et al. Tacrolimus versus mycophenolate mofetil for induction therapy of lupus nephritis: A randomised controlled trial and long-term follow-up. Ann Rheum Dis. 2016;75(1):30-36

29. Liu Z, Zhang H, Liu Z, et al. Multitarget therapy for induction treatment of lupus nephritis. Ann Intern Med. 2015;162(1):18.

30. Liu B, Ou Q, Tang Y, et al. Corticosteroids combined with doublet or single-agent immunosuppressive therapy for active proliferative lupus nephritis. Clin Rheumatol. 2019;38(9):2519-2528.

31. Contreras G, Pardo V, Leclercq B, et al. Sequential therapies for proliferative lupus Nephritis. N Engl J Med. 2004;350(10):971-980.

32. Houssiau FA, D'Cruz D, Sangle S, et al. Azathioprine versus mycophenolate mofetil for long-term immunosuppression in lupus nephritis: results from the MAINTAIN Nephritis Trial. Ann Rheum Dis. 2010;69(12):2083-2089.

33. Dooley MA, Jayne D, Ginzler EM, et al. Mycophenolate versus azathioprine as maintenance therapy for lupus nephritis. N Engl J Med. 2011;365(20):1886-1895

34. Moroni G, Doria A, Mosca M, et al. A randomized pilot trial comparing cyclosporine and azathioprine for maintenance therapy in diffuse lupus nephritis over four years. Clin J Am Soc Nephrol. 2006;1(5):925-932.

35. Chen W, Liu Q, Chen W, et al. Outcomes of maintenance therapy with tacrolimus versus azathioprine for active lupus nephritis: A multicenter randomized clinical trial. Lupus. 2012;21(9):944-952

36. Zhang $\mathrm{H}$, Liu Z, Zhou $\mathrm{M}$, et al. Multitarget therapy for maintenance treatment of lupus nephritis. J Am Soc Nephrol. 2017;28(12):3671-3678.

37. Cattran DC, Feehally J, Cook HT, Liu Z-H, Fervenza FC, Mezzano SA, Floege J, Nachman PH, Gipson DS, Praga M, Glassock RJ, Radhakrishnan J, Hodson EM, Rovin BH, Jha V, Troyanov S, Li PK-T, Wetzels JFM, Cattra JFM. Kidney disease: Improving global outcomes (KDIGO) glomerulonephritis work group. KDIGO clinical practice guideline for glomerulonephritis. Kidney Int Suppl. 2012;2(2):139-274.

38. Moroni G, Gatto M, Raffiotta F, et al. Can we withdraw immunosuppressants in patients with lupus nephritis in remission? An expert debate. Autoimmun Rev. 2018;17(1):11-18.

39. Moroni G, Longhi S, Giglio E, Messa P, Ponticelli C. What happens after complete withdrawal of therapy in patients with lupus nephritis. Clin Exp Rheumatol. 2013;31(78):S75-81.

40. Ward F, Bargman JM. Membranous lupus nephritis: the same, but different. Am J Kidney Dis. 2016;68(6):954-966

41. Austin HA, Illei GG, Braun MJ, Balow JE. Randomized, controlled trial of prednisone, cyclophosphamide, and cyclosporine in lupus membranous nephropathy. J Am Soc Nephrol. 2009;20(4):901-911.

42. Yu F, Haas M, Glassock R, Zhao M-H. Redefining lupus nephritis: Clinical implications of pathophysiologic subtypes. Nat Rev Nephrol. 2017;13(8):483-495.
43. Radhakrishnan J, Moutzouris DA, Ginzler EM, et al. Mycophenolate mofetil and intravenous cyclophosphamide are similar as induction therapy for class $v$ lupus nephritis. Kidney Int. 2010;77(2):152-160.

44. Mok CC, Ying KY, Yim CW, Ng WL, Wong WS. Very long-term outcome of pure lupus membranous nephropathy treated with glucocorticoid and azathioprine. Lupus. 2009;18(12):1091-1095.

45. Chavarot N, Verhelst D, Pardon A, et al. Rituximab alone as induction therapy for membranous lupus nephritis. Medicine (Baltimore). 2017;96(27):e7429.

46. Lee YH, Song GG. Comparative efficacy and safety of tacrolimus, mycophenolate mofetil, azathioprine, and cyclophosphamide as maintenance therapy for lupus nephritis: A Bayesian network meta-analysis of randomized controlled trials. Z Rheumatol. 2017;76(10):904-912.

47. Bertsias GK, Tektonidou M, Amoura Z, et al. Joint European League Against Rheumatism and European Renal Association-European Dialysis and Transplant Association (EULAR/ERA-EDTA) recommendations for the management of adult and paediatric lupus nephritis. Ann Rheum Dis. 2012;71(11):1771-1782

48. Weidenbusch M, Römmele C, Schröttle A, Anders H-J. Beyond the LUNAR trial. Efficacy of rituximab in refractory lupus nephritis. Nephrol Dial Transplant. 2013;28(1):106-111.

49. Rovin BH, Ayoub I. MAINTAINing perspective in lupus nephritis trials. Nat Rev Nephrol. 2015;11(7):389-390.

50. Lenz $\mathrm{O}$, Waheed A a, Baig A, Pop A, Contreras G. Lupus nephritis: maintenance therapy for lupus nephritis-do we now have a plan? Clin J Am Soc Nephrol. 2013;8(1):162-171.

51. Brunner HI, Bennett MR, Abulaban K, et al. Development of a novel renal activity index of lupus nephritis in children and young adults. Arthritis Care Res (Hoboken). 2016;68(7):1003-1111.

52. Martínez-Martínez MU, Llamazares-Azuara LMDG, Martínez-Galla D, et al. Urinary sediment suggests lupus nephritis histology. Lupus. 2017;26(6):580-587.

53. Hebert LA, Dillon JJ, Middendorf DF, Lewis EJ, Peter JB. Relationship between appearance of urinary red blood cell/white blood cell casts and the onset of renal relapse in systemic lupus erythematosus. Am J Kidney Dis. 1995;26(3):432-438.

54. Rhee RL, Davis JC, Ding L, et al. The utility of urinalysis in determining the risk of renal relapse in ANCA-associated vasculitis. Clin J Am Soc Nephrol. 2018;13(2):251-257.

55. Malvar A, Pirruccio P, Alberton V, et al. Histologic versus clinical remission in proliferative lupus nephritis. Nephrol Dial Transplant. 2017;32(8):1338-1344.

56. De Rosa M, Azzato F, Toblli JE, et al. A prospective observational cohort study highlights kidney biopsy findings of lupus nephritis patients in remission who flare following withdrawal of maintenance therapy. Kidney Int. 2018;94(4):788-794.

57. Malvar A, Alberton V, Lococo B, et al. Kidney biopsy-based management of maintenance immunosuppression is safe and may ameliorate flare rate in lupus nephritis. Kidney Int. 2019;1-7.

58. Kaiser R, Cleveland CM, Criswell LA. Risk and protective factors for thrombosis in systemic lupus erythematosus: Results from a large, multi-ethnic cohort. Ann Rheum Dis. 2009;68(2):238-241.

59. Alarcón GS, McGwin G, Bertoli AM, et al. Effect of hydroxychloroquine on the survival of patients with systemic lupus erythematosus: Data from LUMINA, a multiethnic US cohort (LUMINA L). Ann Rheum Dis. 2007;66(9):1168-1172.

60. Sisó A, Ramos-Casals M, Bové A, et al. Previous antimalarial therapy in patients diagnosed with lupus nephritis: Influence on outcomes and survival. Lupus. 2008;17(4):281-288.

61. Kasitanon N, Fine DM, Haas M, Magder LS, Petri M. Hydroxychloroquine use predicts complete renal remission within 12 months among patients treated with mycophenolate mofetil therapy for membranous lupus nephritis. Lupus. 2006;15(6):366-370.

62. Tsakonas E, Joseph L, Esdaile JM, et al. A long-term study of hydroxychloroquine withdrawal on exacerbations in systemic lupus erythematosus. Lupus. 1998;7(2):80-85

63. Broder A, Khattri S, Patel R, Putterman C. Undertreatment of disease activity in systemic lupus erythematosus patients with endstage renal failure is associated with increased all-cause mortality. J Rheumatol. 2011;38(11):2382-2389.

\section{Correspondence to:}

David Navarro, MD

Centro Hospitalar Universitário Lisboa Central E.P.E., Hospital Curry Cabral,

Rua da Beneficência 8, 1069-166 Lisbon, Portugal

E-mail: Davidbnavarro@gmail.com 